\title{
ITINÉRAIRES CULTURELS MODERNES ET CONTEMPORAINS
}

Colportage et lecture populaire. Imprimés de large circulation en Europe, xvi ${ }^{e}$ XIX ${ }^{e}$ siècles. Sous la dir. de Roger ChARTIER et de Hans-Jürgen LüSEBRINK. Paris, IMEC Éditions, Éd. de la Maison des sciences de l'homme, 1996. $17 \times 23,5$, 471 p., ill. (In Octavo).

Le problème de la lecture «populaire », du colportage, de la diffusion des livres destinés à un plus large public et des pratiques qui leur sont liées, a été l'un des plus agités par l'historiographie française voici presque un quart de siècle; il suffit de se reporter au travail de Jean-Jacques DARMON, Le Colportage de librairie sous le Second Empire (Paris, Plon, 1972), élargi et renouvelé par Laurence Fontaine, Histoire du colportage en Europe, $X V^{e}$-XIX ${ }^{e}$ siècle (Paris, Albin Michel, 1993), et à la vaste bibliographie sur la «Bibliothèque bleue », depuis le recensement de Morin et les études de G. Bollème, Robert Mandrou, L. Andriès, etc. Une première mise au point proposée par Henri-Jean Martin («Culture écrite et culture orale, culture savante et culture populaire dans la France d'Ancien Régime », Journal des savants, $3-4,1975$, p. 225-282) avait heureusement clarifié les termes du débat, tandis que les développements de la réflexion sur la mise en texte ouvraient des perspectives très neuves et encore trop négligées (Mise en page et mise en texte du livre manuscrit, dir. Henri-Jean Martin et Jean Vezin, Paris, Éd. du Cercle de la librairie, 1990). Le colloque tenu à Wolfenbüttel en 1991, et dont les Actes paraissent enfin, offre à tout le moins l'occasion d'un état des lieux toujours utile, surtout dans une perspective d'histoire comparée.

Il n'est pas possible de présenter, même sommairement, un synopsis de la vingtaine de contributions diverses et souvent riches qui constituent l'ouvrage. Elles s'organisent autour de trois thèmes principaux successifs ("Usages et réceptions », «Genres : structures et évolutions », «Aires culturelles nationales et modes de circulation transnationaux ») : ce choix interdit, pratiquement, de tirer des conclusions nettes d'une évolution qui conduit le lecteur de la révolution gutenbergienne à la seconde révolution du livre, celle de l'industrialisation. Une relecture du volume permet en revanche d'en reconstruire le contenu en fonction d'axes transversaux plus particulièrement porteurs.

Le premier est, indiscutablement, celui de la chronologie, qui se définit indirectement comme une chronologie politique. Plusieurs des contributions insistent sur la rupture observée, dans les définitions des genres littéraires et dans les usages qui les sous-tendent, à partir des années 1760 , rupture qui se prolonge tout au long du 
XIX ${ }^{e}$ siècle. L'invention de la presse périodique à grand tirage change radicalement, en Occident, les catégories de la littérature populaire, comme le souligne notamment Jean-François Botrel à propos de «la littérature de cordel en Espagne» (p. 278), tandis que la poussée sur les structures de diffusion explique, à la même époque, la multiplication des procès engagés, au Portugal, entre libraires «établis » et nouveaux venus (Diogo Ramada Curto, p. 302). Enfin, comme le montre Lodovica Braida, le genre de l'almanach subit alors des métamorphoses fondamentales (p. 200) : et de souligner comment cette évolution renvoie, plus en profondeur, à l'élargissement des publics, donc à la mise en œuvre progressive de nouveaux critères de la distinction littéraire (une redéfinition du «populaire », selon un thème qui revient à plusieurs reprises dans le recueil).

Il faut ajouter que, même si Alexandre Dutu insiste (p. 165 sq.) sur l'importance de la diffusion par le biais du manuscrit au XvIII ${ }^{e}$ siècle, on regrette l'absence de mise en perspective en amont : l'invention de la typographie en caractères mobiles est implicitement présentée comme une rupture radicale, le problème de la diffusion antérieure de type de textes «populaires » n'est pas évoqué — qu'il s'agisse de manuscrits, voire même de livrets xylographiques ou d'images (voir Fifteenthcentury italian woodcuts from Bibliotheca Classense in Ravenna, Ravenne, 1989). Une partie de la problématique, qui permettrait d'inscrire le problème du colportage et de la lecture populaire dans le temps long de l'histoire moderne, est ainsi malheureusement passée sous silence.

Cette chronologie renvoie à une généalogie des textes abordée par des études sur des textes comme le Fortunatus, le roman de Grisélidis ou celui de Geneviève de Brabant (p. 399); elle n'est pas univoque, mais elle débouche sur une double opposition, et, d'abord, sur la mutation des formes matérielles des objets et des pratiques qui les sous-tendent. Reprenant le problème de la circulation de l'information sous la Révolution française, Hans-Jürgen Lüsebrink et R. Reichardt rappellent, par exemple, que l'époque voit le changement en profondeur des supports et des mises en texte: la catéchèse révolutionnaire vise la participation de tous à la chose publique, elle s'appuie sur la circulation élargie des «connaissances utiles, jusqu'aux dernières ramifications de la société » (p. 80), et donc sur l'adaptation des formes et des messages. Louis-Sébastien Mercier, écrit : (Le Nouveau Paris, Paris, Fuchs, Pougens \& Cramer, an VIII, 6 vol., ici nouv. éd. Paris, Mercure de France, 1995, t. V, p. 208). « Autrefois, les affiches n'apprenoient tout au plus au public que la vente des maisons de campagne [...]. Aujourd'hui, [elles] forment un cours de morale, de politique et de littérature... ». D'autres évolutions sont étudiées dans le recueil, par exemple sur le problème de la vulgarisation scientifique (p. 173 sq.), ou encore sur le contenu des almanachs italiens (p. 183 sq.), suisses-allemands (p. 209 $s q$.), allemands (p. $239 s q$.), etc. Il aurait d'ailleurs été intéressant de reprendre, sous cet aspect, la problématique de la Réforme luthérienne, dont on a pu montrer que le succès était directement lié à l'invention et à la diffusion en masse d'imprimés d'un type nouveau (y compris des images, notamment des caricatures, etc.). Les problèmes de la réception sont cependant surtout abordés par le biais, classique, de la Rezeptionsgeschichte allemande.

Enfin, cette approche débouche sur une géographie différenciée de la diffusion, des pratiques et des usages, géographie présente dans le recueil de manière quelque 
peu implicite, ou par le biais particulier des «modes de circulation transnationaux » (p. $271 s q$.). À cet égard, la problématique des «transferts culturels» pourrait se révéler porteuse (voir Michel Espagne et Michael Werner, « Deustch-französischer Kulturtransfer als Forschungsgegenstand. Eine Skizze », in Transferts. Les relations interculturelles dans l'espace franco-allemand (XVIII ${ }^{e}$-XIX ${ }^{e}$ siècle), Paris, Recherche sur les civilisations, 1988, p. 11-34). Une première rupture oppose, dans tous les cas, la ville, pôle de l'innovation et de la modernité, au plat-pays (voir La Ville et la diffusion de l'innovation en Europe occidentale, XIV ${ }^{e}$-XIX ${ }^{e}$ siècle, dir. Bernard Lepetit et Jochen Hoock, Paris, École des hautes études en sciences sociales, 1987) : non seulement la ville structure, matériellement, les réseaux de la distribution, mais elle est également le lieu où s'élaborent le discours des élites, la définition du populaire et, le cas échéant, la politique que l'on mettra en œuvre pour favoriser l'acculturation du plus grand nombre. D'autres ruptures s'organisent en arrière-plan : celle, pour l'essentiel passée sous silence dans le volume, entre pays catholique et pays de réforme, celle aussi entre l'ancienne Europe de l'écrit et de l'imprimé (l'Europe rhénano-mosane, l'Italie du Nord, les Flandres) et les territoires de plus en plus vastes progressivement intégrés par le modèle occidental. L'imprimé joue un rôle décisif dans le processus : après l'effondrement des $\mathrm{XVI}^{\mathrm{e}}$ et $\mathrm{XVII}{ }^{\mathrm{e}}$ siècles, le développement du nouvel empire des Habsbourg s'appuie sur la réorganisation progressive des réseaux du livre, et sur la diffusion systématiquement organisée par l'administration de Vienne, de livres scolaires et d'imprimés de grande diffusion susceptibles de faciliter le passage à la modernité culturelle - ainsi dans le cas de la Hongrie, abordée par la contribution de Marta Gelleri-Lazar.

Bien entendu, cette problématique débouche à nouveau, à partir de la seconde moitié du XVIII et surtout au XIX ${ }^{\mathrm{e}}$ siècle, sur la problématique de la seconde révolution du livre et des nationalités : le statut et le rôle des imprimés de plus grande diffusion s'en trouvent déplacés le plus en profondeur - comme le montre l'exemple des almanachs allemands, étudiés par Y.-G. Mix. Il est très important de repérer, de décrire et de réinscrire dans les temporalités les plus larges l'histoire des imprimés les plus communs à l'instant même où ils connaissent leurs mutations les plus profondes. L'invention du folklore a déjà déplacé le statut du texte, comme le souligne Heinrick Heine dans De l'Allemagne (voir trad. franç. Paris, 1981, p. 214) déjà à la recherche de l'authenticité disparue: "Quelque belle que soit la Geneviève de M. Tieck, j'aime mieux le livre populaire, mal imprimé à Cologne sur le Rhin, avec de mauvaises gravures en bois où l'on a représenté la pauvre princesse palatine toute nue, chastement couverte de ses longs cheveux, et faisant allaiter son enfant par une biche compatissante [...]. »

Et, à la fin du siècle, à Combray (voir Marcel Proust, Du côté de chez Swann, nouv. éd. Paris, 1973, p. 9-10) si Geneviève est toujours présente, c'est seulement, en manière de dernier avatar, par l'intermédiaire de la lanterne magique et des peintures sur verre projetées sur les murs de la chambre du narrateur : «Golo s'arrêtait un instant pour écouter avec tristesse le boniment lu à haute voix par ma grand'tante [...]. Puis il s'éloignait du même pas saccadé. Et rien ne pouvait arrêter sa lente chevauchée [...]. » 
Histoire et recueil des Lazzis. Éd. critique par Judith Curtis et David TRotT. Oxford, Voltaire Foundation, 1996. $16 \times 24$, vIII-272 p., bibliogr., index (Studies on Voltaire and the Eighteenth Century, 338).

Judith Curtis et David Trott publient un manuscrit du XvIII siècle, intitulé « Histoire et recueil de Lazzis »; il s'agit d'un ensemble de petites pièces, parades, saynètes, chansons, conçu pour la distraction raffinée d'un cercle privé, qui réunit des comédiennes et la fine fleur de l'aristocratie. Mademoiselle Quinault reçoit ainsi le comte de Livry, le comte de Caylus et le ministre Maurepas. Une microsociété de huit personnes, qui se retrouvent régulièrement dans les années 1731-1732, et agrémentent leurs soupers de représentations théâtrales privées.

Le document ici reproduit permet de constater l'importance du théâtre de société au début $\mathrm{du} \mathrm{XVIII}^{\mathrm{e}}$ siècle, importance qui, selon Curtis et Trott, a été sous-estimée, faute d'archives. Le comte de Caylus, qui commente les différentes représentations, indique comment elles ont été préparées, exécutées et reçues, imite à son insu Marivaux : "Alors que Caylus fait de la société des Lazzistes un prétexte pour des réflexions sur le théâtre (rejoignant le groupe encore restreint dans les années 1730 des historiens et théoriciens de la représentation), Marivaux va intensifier son utilisation du théâtre pour faire des réflexions sur la société, et en particulier sur le langage des salons [...]. »

La présente et savante édition comporte une introduction analytique, des notes, un index, une bibliographie, elle permet de mieux comprendre le fait théâtral au $\mathrm{XVIII}^{\mathrm{e}}$ siècle dans toute sa riche diversité, et elle saisit sur le vif une forme de sociabilité qui rapproche pour un temps et abolit les distances entre comédiens, comédiennes et grands seigneurs.

Monique COTTReT

Le Groupe de Coppet et le monde moderne. Conceptions, images, débats. Actes du $\mathrm{VI}^{\mathrm{e}}$ colloque de Coppet organisé par la société des Études staëliennes (Paris) et l'association Benjamin-Constant (Lausanne), Liège, 10-12 juil. 1997, publ. par Françoise TiLkin. Liège/Genève, Bibliothèque de la faculté de philosophie et lettres de l'université de Liège/Diff. Droz, 1998. $16 \times 24$, 444-16 p., index (Bibliothèque de la faculté de philosophie et lettres de l'université de Liège, fasc. CCLXXVII).

Heinrich Heine, dans le portrait caricatural qu'il a esquissé de Germaine de Staël, écrivait que la « sultane de la pensée » était partie en Allemagne résolue d'y découvrir « un nébuleux pays d'esprits, où des hommes sans corps et tout vertu se promènent 
sur des champs de neige, ne s'entretenant que de morale et de métaphysique ». Promouvoir le spiritualisme allemand pour l'opposer au matérialisme français (incarné, d'après madame de Staël, par Napoléon), voilà en quoi se résumaient, à en croire Heine, les motifs qui amenèrent l'hôte de Coppet à tant d'éloges de la pensée allemande et à tant d'aveuglement face à la réalité d'outre-Rhin.

Cependant, s'il y a du vrai, malgré son parti pris explicite, dans la description de Heine, il n'en reste pas moins que le De l'Allemagne, tout comme l'ensemble de l'œuvre de l'hôte de Coppet, est indispensable pour la compréhension de la production intellectuelle du XIX ${ }^{\mathrm{e}}$ siècle français. L'apport des colloques de Coppet, dont la tradition remonte à 1966, est, de ce point de vue, décisif pour l'historien des idées : grâce à l'étude de ce carrefour intellectuel que fut Coppet, on connaît mieux maintenant, non seulement les œuvres, prises séparément, des auteurs qui en font partie mais aussi et surtout le contexte dans lequel ces œuvres s'inscrivent et leurs interférences. Voici donc les actes de ce $\mathrm{VI}^{\mathrm{e}}$ colloque de Coppet (avec vingt-deux communications, divisées en six séances de travail), dont le principal défaut est de ne s'intéresser pratiquement qu'à madame de Staël et à Benjamin Constant (ce que François Rosset n'oublie pas, d'ailleurs, de noter dans ses propos conclusifs). En effet, il faudra presque attendre la dernière communication du colloque, celle de John Isbell, pour apprendre qu'à ce groupe appartenaient également les frères August Wilhelm et Friedrich von Schlegel, J. Ch. L. Sismondi, Camille Jordan, Mathieu Montmorency-Laval, madame Récamier ou Charles de Bonstetten. L'approche d'Isbell est intéressante non seulement parce qu'on y retrouve enfin le groupe, mais aussi et surtout parce qu'il entreprend de retracer le cheminement intellectuel et politique des représentants de Coppet au moment de l'avènement, sous la Restauration, du romantisme libéral; ce qui permet la formulation de questions fécondes, comme celle de l'homogénéité du groupe au-delà de Coppet, le problème des successeurs - dans le sens métaphorique aussi bien que littéral du terme —, l'impact éditorial des idées de Coppet sur l'opinion publique de cette période ou encore la question des liens établis entre les survivants de Coppet et les doctrinaires, les globistes, les ultras.

Le confinement aux seuls protagonistes de Coppet qui caractérise le volume dans son ensemble se trouve d'une certaine manière compensé par la richesse et la variété des approches (inévitablement hétéroclites du point de vue méthodologique) qui ont amené plusieurs participants soit à creuser dans des écrits relativement peu connus de Benjamin Constant et de madame de Staël soit à explorer des thématiques originales issues de leurs écrits. Tel est le cas des communications de Roger Francillon («Benjamin Constant et le jeu»), Sabina Kruszynska (« De la religion... de Benjamin Constant. Quelques aspects philosophiques»), Jan Herman («"Ton regard m'apprendra tes plus secrètes pensées". La rhétorique de la distance dans Delphine »), Avriel H. Goldberger («De Delphine à Corinne, sous la loupe du traducteur $\gg)$.

Notre attention a été davantage retenue par la communication de Gérard Gengembre et Jean Goldzink ( « Madame de Staël ou pour une religion politique ») où, à partir des quelques pages consacrées à la religion dans les Circonstances actuelles, les deux auteurs reconstituent la problématique staëlienne sur la place qu'est censé occuper le religieux dans une république moderne et, partant, philosophique. Le 
concept de tolérance comme «critère absolu» de la conception religieuse de madame de Staël est ici finement analysé, et on comprend bien de quelle manière a évolué le penchant staëlien pour le protestantisme au fil de ses écrits ultérieurs. Enfin, à travers le rapprochement heureux entre madame de Staël et l'œuvre de son père, Jacques Necker, se profile le caractère opératoire de l'imbrication chez madame de Staël des principes et des circonstances, "de l'immuable et du moment » (p. 219). Ce sont les mêmes circonstances qui l'ont amenée d'ailleurs à une réévaluation du concept de «bonheur», analysé par Kristin Anders («Les visions du bonheur dans une société moderne chez madame de Staël »), réévaluation qui marque une nette rupture avec les sens dont ce concept avait été investi par les Lumières. De ce même point de vue, le texte de Jacques Dubois («Madame de Staël et l'institution littéraire ») met en relief la nouvelle tâche que donne à l'écrivain l'œuvre, sociologique avant la lettre, de madame de Staël, lorsqu'elle l'appelle à assurer «l'interaction entre le peuple et ses représentants » (p. 37). En interrogeant les modalités de la perfectibilité, notion qui structure de part en part les lignes de force ainsi que les limites du libéralisme constantien, Florence Lotterie (« Le progrès désenchanté. La perfectibilité selon Constant ou le malaise libéral ») renouvelle la discussion sur ce concept, légué par le XVIII ${ }^{\mathrm{e}}$ siècle, sensiblement remanié au cours $\mathrm{du} \mathrm{XIX}^{\mathrm{e}}$ et qui a fini par devenir le moteur canonique de l'interprétation historique.

La contribution de Lucia Omacini et Roswitha Schatzer ("Quand Benjamin Constant travaille sur les papiers de madame de Staël. Le cas de la "Copie" des Circonstances actuelles ») nous donne en détail, dans un tableau très riche et rigoureux, dont la lecture est facilitée par des remarques subtiles, les passages de la «Copie» des Circonstances actuelles repris par Constant dans ses Fragments d'un ouvrage abandonné et dans ses Principes de politique.

En lisant les actes de ce colloque on se rend compte aussi de l'impact croissant des gender studies, avec les communications de Claire Jaquier ( Le féminin et le masculin. Une redistribution des cartes ») et de Christine Planté («Passion et création, l'insoluble conflit des héroïnes staëliennes. Corinne et Sapho»); on voit encore l'intérêt que suscite toujours la question de l'implication du sujet dans les récits du $\mathrm{XIX}^{\mathrm{e}}$ siècle, avec l'intervention de Jean-Marie Roulin («Temporalité et construction du sujet dans les écrits de Constant») et celle d'Anne Amend-Söchting ( " "Analyse du moi" — "Culte du moi”. Benjamin Constant et Maurice Barrès »). On se rend compte enfin de l'actualité de la question de l' «opinion publique » et de son autorité qui n'a cessé de croître depuis la Révolution, et l'on mesure l'importance de la redéfinition des rapports pouvoir/savoir, discours/action avec les articles de Susan Tenenbaum («Public opinion and the modern state»), Beatrice Fink («Benjamin Constant. Mobilisation et médiatisation du mot») et Karyna Szmurlo ( Vers la théorie du performatif. Germaine de Staël et les discours de la Révolution»).

En fait, c'est autour de la notion centrale de «monde moderne» et de ses différentes acceptions que s'organisent les orientations de ce colloque. On peut aisément distinguer, à cet égard, au moins trois pistes clairement définies. Tout d'abord, pour un certain nombre de communications parmi celles que nous avons déjà citées, le «monde moderne » est celui qui surgit de la coupure avec le «monde ancien », celui de l'Ancien Régime. 
Ensuite, à en croire certains des intervenants, il semblerait que le «monde moderne » de Coppet inclut la modernité libérale, voire néolibérale, $\mathrm{du} \mathrm{xx}^{\mathrm{e}}$ siècle, et peut ainsi être questionné sous une lumière tout actuelle. Dans ce contexte, Lucien Jaume («Le problème de l'intérêt général dans la pensée de Benjamin Constant ») nous invite, par exemple, à « redécouvrir, avec le groupe de Coppet, que le libéralisme est, dans son moment de naissance, une forme de culture morale et d'éducation de l'individu» (p. 176). En réhabilitant le libéralisme constantien, minoritaire, voire « hérétique », tel qu'il émerge, plus précisément, des réflexions de l'auteur sur l'intérêt général, Jaume relève ce qui sépare Constant non seulement de Cabanis mais aussi de Sieyès et de l'utilitarisme anglais, et montre la manière dont Constant, dans sa tentative de trouver une réponse à la question de l'Unité qui hante le discours révolutionnaire, est porté à faire une distinction subtile entre «intérêt général » et « intérêt de tous », et à forger, au passage, une conception originale du fédéralisme. Mauro Barberis ( Constant, madame de Staël et la constitution républicaine. Un essai d'interprétation ») reprend, de son côté, le débat sur l'évolution du constitutionnalisme chez les deux auteurs; à la lumière des analyses de Friedrich A. von Hayek sur les traditions opposées du libéralisme «évolutionniste » anglais et du libéralisme «constructiviste » français, Barberis soutient dans la dernière partie de son intervention que Constant réalise, sous la Restauration, une ouverture sur le modèle libéral anglais.

Enfin, une troisième acception de la notion de «monde moderne » a amené à réfléchir sur le couple antithétique Anciens/Modernes, qui est au centre d'une des discussions les plus passionnées chez les représentants de Coppet. Jean-Pierre Perchellet («De l'extériorité antique à l'intériorité romantique. Madame de Staël et le modèle tragique grec $»)$ présente les conceptions littéraires de madame de Staël telles qu'elles s'éclairent par sa critique du classicisme français et des règles aristotéliciennes; en effet, Perchellet l'a bien vu, c'est sur l'opposition extériorité (du modèle ancien, et donc de la littérature française du XvII ${ }^{\mathrm{e}}$ siècle)/intériorité (celle des Modernes, qui se doit de devenir romantique) que se fonde toute la réflexion staëlienne. Cependant, le couple corollaire «fixité/mobilité » ne devrait-il pas être rapproché de l'idée de progrès chère à madame de Staël, qui ne cesse de rappeler l'ignorance des Anciens en la matière? Dans cette même perspective, Ephraïm Harpaz («Benjamin Constant entre les Anciens et les Modernes. Orientations polyvalentes ») essaie de mesurer l'attachement de Constant à l'érudition antique et nous offre quelques pages saisissantes sur le De la religion (voir aussi l'article de Sabina Kruszynska), en replaçant cette œuvre, en apparence extra-politique, dans le contexte politique qui lui est propre.

Chryssanthi AvLami

Philologiques IV. Transferts culturels triangulaires France-Allemagne-Russie. Sous la dir. de Katia Dmitrieva et de Michel Espagne. Paris, Éditions de la Maison des sciences de l'homme, 1996. $15 \times 22$, 435 p., index.

Le propos est d'analyser les «interactions des trois "aires culturelles" » que constituent la France, l'Allemagne et la Russie telles que peuvent les saisir « l'ethno- 
logue, l'historien de la littérature et de la philosophie ». La période d'étude s'étend du milieu du XVIII ${ }^{e}$ siècle à la révolution de 1917, et les différents articles qui composent le livre sont l'œuvre de chercheurs francophones, russes et allemands.

L'ouvrage est articulé en quatre moments. Le premier, «Palimpsestes », s'attache aux «superpositions des trois strates culturelles », le deuxième à «l'antagonisme des paradigmes », un troisième moment traite des stéréotypes, viennent enfin des textes qui exposent différents parcours intellectuels intéressant les trois cultures.

L'article d'Ewa Bérard, consacré à Saint-Pétersbourg, ville des Lumières, ouvre le premier moment. Il nous montre que les représentations de cette ville en Allemagne comme en France résultent moins des récits des voyageurs et de la propagande russe que des espoirs et des inquiétudes qui habitent les philosophes occidentaux eux-mêmes. La figure d'August Ludwig von Schlözer (1735-1809) est évoquée par Antonia Bernard. L'œuvre majeure de ce philologue et historien allemand est l'établissement d'une première édition critique rigoureuse et d'une traduction de la «Chronique de Nestor». Celle-ci devait servir de référence à l'édition russe puis française du texte. C'est Schlözer qui sut dénoncer en Voltaire, auteur de l'Histoire de l'Empire de Russie sous Pierre le Grand, le «laudateur stipendié d'un grand tsar » (Peter Hoffmann, Gabriela Lehmann-Carli). Bref, l'action de Schlözer a largement favorisé le développement de la connaissance du passé russe en Allemagne, en France comme en Russie elle-même. Enfin, Efim Edkind met au jour la conjonction des modèles français et allemands dans l'œuvre du poète Gavrila R. Derjavine (1743-1816) qui semble donner déjà raison à ces vers que Alexandre A. Blok écrira près de cent cinquante ans plus tard : "Tout nous est proche : la finesse de Gaulois,/ Et l'obscur génie du Germain [...]. »

L' « antagonisme des paradigmes » se manifeste dans des figures comme celles de Herder et de Tourgueniev. Le premier place ses espoirs européens, tantôt dans la France, tantôt dans la Russie (Pierre Pénisson); l' «impartialité cosmopolite » du second le conduit à une égale hostilité à l'égard des nationalismes français et allemands naissants (Vera Miltchina). Frances Nethercott s'efforce de mesurer les influences antagonistes des institutions françaises et allemandes dans l'établissement du système scolaire russe. L'accent mis sur la philologie classique marque la prédominance du modèle germanique : grammaires et manuels sont souvent traduits de l'allemand.

En France, le stéréotype du Russe fut longtemps le Cosaque, personnage complexe, généralement symbole de barbarie, parfois d'énergie et de rébellion, comme pour Pierre-Joseph Proudhon. Ultérieurement, l'alliance contre l'ennemi commun allemand vint transformer, à la veille de la Grande Guerre, le «mangeur de chandelles » en figure providentielle, soudain investie de toutes les qualités qui lui faisaient défaut (Galina Kabakova, dont l'article est illustré de plusieurs reproductions d'images populaires). Nicolaï Iaremenko montre que la référence négative aux stéréotypes de l'Allemand et du Français fut un élément décisif dans la formation de l'identité nationale russe.

Michel Cadot décrit le parcours qui conduit, au XIX ${ }^{\mathrm{e}}$ siècle, l'intelligentsia russe de l'idéalisme allemand au socialisme à la mode française, en s'attachant tout particulièrement à l'exemple de Mikhaïl Bakounine et d'Alexandre Herzen : « Hegel et vous, vous avez fait la moitié de mon éducation philosophique », écrivait ce dernier 
à Proudhon. Michel Espagne s'attache aux relations culturelles franco-germanorusses entre 1870 et 1914 , et il démontre que, en dépit des clivages politiques qui marquent la période et des ruptures apparentes, on assiste en vérité à « une interpénétration croissante des trois cultures ». Katia Dmitrieva et Frances Nethercott exposent la fusion des héritages nietzschéens et bergsoniens dans la pensée russe du début de ce siècle. Enfin, Jean-Pierre Morel s'attache à l'analyse d'un «épisode prolétarien » qui a marqué la littérature des trois pays durant les années 1920.

La réunion de ces articles et de quelques autres présente le très grand intérêt de mettre en lumière l'action décisive de personnages méconnus et surtout de donner toute leur dimension à des mouvements européens dont on restreint généralement l'examen au cadre national ou en rapprochant tout au plus deux pays.

L'ampleur du champ d'étude, l'étendue de la période et la multiplicité des réalités que peut prétendre embrasser le terme de «culture », s'ils offrent de nombreux objets d'analyse et permettent une grande diversité d'approches, rendent une synthèse fort difficile. Mais bien que les différents textes fassent en effet apparaître un faisceau extrêmement complexe de relations, ils laissent néanmoins entrevoir certaines constantes, certaines articulations chronologiques dont l'exposé conclusif aurait sans doute encore enrichi l'ouvrage.

Igor SOKOLOGORSKY

Isabelle OLIVERo, L'Invention de la collection. De la diffusion de la littérature et des savoirs à la formation du citoyen au XIX ${ }^{e}$ siècle. Paris, Institut Mémoires de l'édition contemporaine/Maison des sciences de l'homme, 1999. $17 \times 23,5$, 336 p., index (In Octavo).

Ainsi, les collections ont une histoire. Le $\mathrm{xIX}^{\mathrm{e}}$ siècle, siècle du roman, de l'échange épistolaire, du journal intime, est aussi le siècle de la construction d'une mémoire, de la formation d'un savoir par la constitution des « bibliothèques », grâce aux efforts inventifs d'éditeurs-imprimeurs à l'origine du succès des « collections » dont nous entretient Isabelle Olivero dans un ouvrage issu de sa thèse.

Olivero nous le rappelle : «éduquer, instruire, régénérer » fut la « mission civilisatrice » des autorités du XIX ${ }^{\mathrm{e}}$ siècle (p. 267). De l'éducation du peuple à la propagande pour l'instruction, tous ont cherché à élargir le cercle des initiés au savoir historique, scientifique, philosophique et, bien sûr, littéraire.

L'auteur a analysé les collections d'auteurs classiques et contemporains pendant la période d'apprentissage de la démocratie — de 1816 à 1876 —, le catalogue de la bibliothèque Charpentier et celui de la Bibliothèque nationale; de son point de vue, le grand initiateur de l'œuvre fut, sans conteste, Gervais Charpentier qui, en 1838, inventa la «collection moderne ». L'objectif de Charpentier est double : à la fois lutter «contre la contrefaçon» et contre les «cabinets de lecture » où les « lecteurs ne venaient plus tellement pour louer des livres mais pour lire les derniers épisodes des romans à succès ». Novation à la fois technique, commerciale et culturelle, l'édition moderne bouleverse alors les habitudes de la profession en même temps qu'elle révolutionne l'accès à la culture : le lecteur loueur de livres peut, par la 
modicité des prix, se constituer « une bibliothèque de classiques modernes » (p. 70). Dans l'ensemble des ouvrages édités de 1838 à 1871, les écrivains contemporains se taillent la part du lion. "Tandis que Gervais Charpentier édite presque tous les auteurs romantiques français, son fils Georges sera l'éditeur des réalistes et des naturalistes » (p. 118). Parmi les collections ainsi accessibles à un public plus large, il faut noter l'importance accordée à la littérature grecque et, dans une moindre mesure, aux traductions d'auteurs anglais, allemands et italiens.

Après le succès de Louis Hachette qui importe d'Angleterre, «le système des bibliothèques de gare»(p. 71), c'est dans l'imprimerie Dubuisson que naît, en 1863, la collection de classiques de la Bibliothèque nationale. L'association, dont les typographes avaient pris l'initiative, connaît bientôt le succès et l'indépendance en permettant à « la société des éditeurs » de s'affirmer publiquement, à la fin des années 1860, « comme un organe populaire d'émancipation intellectuelle » (p. 102).

Dans la diffusion du savoir, le rôle des préfaciers semble déterminant. C'est ainsi que George Sand « réussit en dix-huit pages à réhabiliter Rousseau parce qu'il est à ses yeux le seul philosophe religieux du XVIII ${ }^{\mathrm{e}}$ siècle » (bibliothèque Charpentier, 1848). Dix-sept ans plus tard, Nicolas David pour la «Bibliothèque nationale » choisit, au contraire, de consacrer Rousseau pour son destin historique.

L'histoire est la grande affaire des collections, l'authenticité des textes anciens comme l'établissement de la vérité historique préoccupent les éditeurs; ainsi, l'Histoire des français depuis le temps des Gaulois jusqu'à nos jours de Théophile Lavallée, publiée en 1844, eut un succès considérable; à la demande de Charpentier, Frédéric Lock, prolongea l'œuvre jusqu'en 1873. «Au total, des livraisons de 32 pages in- 8 à 35 centimes à l'édition illustrée de grand format, également en livraison, ce sont 68000 volumes de ce type qui sont débités entre 1838 et 1848 » (p. 148).

Parmi les auteurs contemporains, les noms les plus fréquemment relevés dans les bibliothèques privées sont ceux de Chateaubriand, madame de Staël, Béranger et Lamartine.

Bien d'autres informations destinées aux spécialistes du XIX ${ }^{\mathrm{e}}$ siècle comme au curieux d'histoire culturelle figurent dans cet ouvrage érudit. Cependant, les conditions de production de ces collections ne sont guère pensées dans leur historicité, le plus souvent à peine esquissée. Le libéralisme du Second Empire, qui prépare à la formation de la III ${ }^{\mathrm{e}}$ République, est bien mis en valeur par la personne d'Édouard Laboulaye, mais son rôle, dans la formation d'un savoir comme dans la constitution d'une mémoire, est trop peu montré. En d'autres termes, il manque une véritable analyse de contenu des ouvrages. On se doit de relever par ailleurs, la méconnaissance de l'histoire des femmes au $\mathrm{XIX}^{\mathrm{e}}$ siècle, ce qui est hélas trop fréquent dans les ouvrages contemporains, malgré une historiographie importante sur le sujet. L'auteur accumule les stéréotypes sur la «littérature féminine». «La» femme aurait un faible goût pour la lecture. Quant au savoir savant, il resterait étranger à la plupart des femmes. Signalons à Isabelle Olivero, la parole de Clémence Robert qui, en 1833, se rebelle contre l'ignorance dans laquelle étaient tenues délibérément les femmes de son temps: "Savoir, c'est vivre et retenir dans l'ignorance, c'est presqu'un homicide. » 
Nikolaus Pevsner, Les Académies d'art. Trad. de l'anglais par Jean-Jacques Bretou, prés. d'Antonio Pinelli, trad. de l'italien par Gisèle D'Antony. Paris, Gérard Monfort, 1999. $17 \times 24,260$ p., index (Imago Mundi).

Traduit en français près de soixante ans après sa publication en 1940, l'ouvrage de Nikolaus Pevsner constitue, aujourd'hui encore, une synthèse inégalée sur l'évolution de l'enseignement artistique entre le $\mathrm{xv}^{\mathrm{e}}$ siècle et le début $\mathrm{du} \mathrm{xx}^{\mathrm{e}}$ siècle en Europe. Comme le souligne le titre original, Academies of art. Past and present, ce livre d'historien est aussi un livre de son temps. Le lieu de première publication, Cambridge, ne doit en effet pas tromper le lecteur. Academies of art a été largement conçu dans l'Allemagne de Weimar, avant que son auteur ne s'exile en Angleterre en 1933, au moment où Walter Gropius fonde le Bauhaus, un mouvement artistique révolutionnaire qui tente d'intégrer les impératifs esthétiques et utilitaires de la société et donc d'instaurer un lien nouveau entre l'artiste et la société industrielle moderne. Pevsner, qui salue cette réconciliation, s'efforce de comprendre les origines historiques du conflit art/société.

Dans cette enquête, les académies d'art jouent un rôle capital puisqu'elles ont contribué à régir les rapports entre l'artiste et la société. Cependant, cette étude institutionnelle ne doit pas être mise en rapport avec une histoire sociale de l'art plus globalisante, et qui reste encore à venir en 1940: «Je ne me suis pas fixé la tâche ambitieuse de procurer cette histoire sociale de l'art qui nous fait si cruellement défaut, afin de remplacer une histoire formelle de l'art; mon travail vient en parallèle. Dans le cas contraire, j'aurais dû inclure l'histoire du goût, l'histoire des théories esthétiques, des expositions, des collections, du marché de l'art. Toutes choses que j'ai laissées de côté, ne m'attachant qu'à un seul aspect : la formation artistique, ou plutôt la formation de l'artiste» (p. 31). Le propos de l'ouvrage est bien de décrire les modes d'enseignement artistique auxquels les emplois successifs du mot «académie» renvoient entre 1500 et 1900 .

Cette étude remarquablement informée est menée de manière chronologique, scandée par des catégories stylistiques (maniérisme, baroque, rococo, néo-classicisme...), dans une perspective historiographique largement influencée par la Geistgeschichte qui explique les caractéristiques historiques d'une période par leur correspondance avec le Zeitgeist. Pevsner souligne ainsi le rôle du maniérisme dans la réglementation des académies d'art : «Il existe un rapport tout aussi évident entre la particularité des nouvelles académies et un autre trait caractéristique du maniérisme : les académies de la Renaissance étaient complètement informelles, celles de l'époque maniériste sont dotées de règles précises et détaillées » (p. 37).

Le chapitre introductif s'attache à montrer la vitalité des premières académies en Italie. Si l'origine grecque du mot «académie» est bien connue, puisqu'elle désignait la communauté que formaient les disciples de Platon, ce terme est remis à l'honneur dès les années 1460, pour désigner l'Accademia Platonica réunie autour de Marsile Ficin. Une académie désigne alors un « cercle informel où se rencontrent des individus unis par le même désir de culture ». Cependant, l'auteur insiste avec force sur la rupture que provoque le maniérisme issu de la Contre-Réforme : les académies du Cinquecento perdent leur caractère informel et leur universalisme en se spécialisant dans des activités littéraires, juridiques, musicales, comme les Umidi de 
Florence (1540) ou les Rozzi de Sienne (1531). Face à cette floraison soudaine d'académies aux appellations nombreuses et aux règles détaillées, le modèle académique se diffuse en Europe du Nord avec la particularité que le terme latin academia y désigne en fait des «universités » où les participants se réunissent autour d'enseignants pour écouter des conférences méthodiques. En Italie, le mouvement est inverse puisque les académies se développent jusqu'à tenir lieu, parfois, d'universités comme la Fiorentina de Florence en 1531.

Durant le Cinquecento, l'académie d'art commence à désigner une école artistique affranchie des contraintes de la corporation. Si Léonard de Vinci apparaît bien comme le précurseur de l'enseignement artistique moderne en défendant un programme révolutionnaire basé sur l'enseignement de la perspective d'abord, puis des proportions, et enfin du dessin, il ne semble pas que son académie ait appliqué cette théorie. C'est en 1563 que naît l'Accademia del Designo à Florence, sous l'impulsion de Giorgio Vasari, dans un contexte de perte d'influence de la corporation: protégée par Côme de Médicis, hiérarchisée et rassemblant les artistes florentins de premier plan, elle est dotée d'une mission éducative. Celle-ci reste cependant secondaire par rapport à l'objectif essentiel de Giorgio Vasari : rompre avec le système médiéval des guildes d'artistes. En revanche, l'Accademia di San Luca à Rome, déclarée ouverte le 14 novembre 1593 à l'initiative du cardinal Borromée et du peintre Federigo Zuccari, a pour but primordial la formation des artistes. L'enseignement du dessin d'après nature et d'après moulages y est fait par des professeurs, qui donnent aussi des conférences sur l'art. L'académie détient le monopole pour l'expertise d'œuvres d'art et alloue occasionnellement des prix. Cependant, les conflits qui marquent les relations entre la corporation et l'académie traduisent la perte de suprématie de cette dernière malgré les réformes d'Urbain VIII. L'apport du Cinquecento est donc essentiel dans l'histoire des académies d'art puisqu'on y voit apparaître la fonction représentative de l'académie qui prépare l'émancipation des artistes par rapport au travail manuel, et sa fonction éducative moderne.

Le Seicento rend compte d'une double évolution, à la fois institutionnelle et sémantique, en Italie. Les académies existantes voient leur fonction progressivement réduite à celle de conseil corporatif, malgré une diffusion du modèle académique dans les autres centres artistiques italiens, comme à Milan. À cet égard, l'auteur met bien en lumière le rôle des personnalités qui ont fait évoluer la situation de l'art en Italie, puisque la mobilité et l'activité de Borromée et de Zuccari traduisent leur apport personnel à la fondation d'académies d'art.

Parallèlement, un glissement sémantique apparaît : au milieu du Seicento, le mot « académie » désigne aussi un cours de dessin d'après modèle vivant, ce qui était reconnu depuis la Renaissance comme l'élément essentiel de la formation artistique; c'est au sujet de l'Accademia degli Incamminati des Carrache à Bologne au début du XVII siècle que l'on trouverait la première trace de cette évolution sémantique.

La diffusion du modèle académique en Europe reste l'acquis essentiel de ce siècle. Aux Pays-Bas, Karel Van Mander essaie d'introduire les innovations italiennes et défend la peinture comme ars liberalis mais se heurte à la vigueur de l'organisation corporative médiévale. L'innovation vient désormais de Paris où l'Académie royale de peinture et de sculpture est ouverte le $1^{\text {er }}$ février 1648 . Pevsner interprète la création du système académique à l'aune du mercantilisme et insiste, sans doute 
exagérément, sur la «volonté consciente [de l'État] de faire perdre aux artistes et amateurs leur indépendance », désormais soumis aux exigences de la cour et dont «l'instabilité sociale » se voit transformée en «statut administratif ingénieusement adapté ». Dans une intéressante tentative d'histoire comparée, Pevsner explique l'échec de l'introduction d'un système académique précoce en Hollande par la différence dans la structuration de la clientèle artistique et dans le statut social des artistes. La demande artistique en France serait soutenue par les commandes d'une clientèle restreinte au fort pouvoir d'achat, tandis qu'en Hollande, il existerait un marché anonyme où les liens entre producteur et consommateur n'existent pas, ce qui favorise une production spécialisée. Cette intuition s'est trouvée à la fois confirmée et nuancée par des travaux récents; ainsi John Michael Montias dans Le Marché de l'art aux Pays-Bas (XV-XVII siècle) montre que les artistes hollandais ont surtout adopté une stratégie mixte où les œuvres sont en partie réalisées à l'avance pour être vendues à un client anonyme, en partie adaptées au goût du client.

Le chapitre consacré à la période néoclassique décrit avec force la floraison nouvelle d'académies d'art dans la deuxième partie du XviII ${ }^{\mathrm{e}}$ siècle (à Vienne, Berlin...) et souligne la pérennité du modèle français. Surtout, Pevsner insiste sur l'importance des considérations économiques : les académies d'art doivent servir à promouvoir le commerce national en diffusant le bon goût parmi les artistes nationaux, de telle sorte qu'ils ne soient pas surpassés par les artistes étrangers. La renaissance de l'académie de Berlin repose ainsi sur son soutien au « développement de l'industrie nationale », et Friedrich Anton von Heinitz demande en 1788 «l'adjonction d'une école destinée à ceux qui comptent exercer un métier ». En ce sens, ce qui distingue essentiellement l'académie de Paris des académies fondées au tournant du siècle est l'introduction de classes professionnelles et de classes élémentaires.

Dans la perspective de l'ouvrage, le $\mathrm{XIX}^{\mathrm{e}}$ siècle apparait comme une parenthèse. En effet, si l'académisation de l'enseignement artistique se fait plus forte à cause de la forte réduction de la formation réglementée en atelier, le romantisme et son « individualisme révolutionnaire » veulent mettre à bas la Bastille académique. Des réformes sont entreprises, et Pevsner se penche longuement sur l'exemple des nazaréens allemands incarnés par Peter Cornelius et Wilhelm Shadow qui essaient, sans succès, de revenir à l'idéal communautaire et fraternel de l'atelier médiéval en créant en 1831 à l'académie de Düsseldorf, des classes magistrales destinées à permettre aux étudiants les plus avancés de louer un atelier dans l'Académie pour une somme modique. Malgré ces tentatives de réforme, la transformation des académies en instituts d'art se développe à cause de la suppression des classes élémentaires, et du relèvement de l'âge requis. Dès lors, les considérations économiques qui avaient si fortement marqué l'évolution du siècle précédent disparaissent.

L'avènement de l'industrie montre alors les effets pervers d'une telle scission entre l'art et la technique. Le déclic vient de l'exposition universelle de 1851 qui révèle la médiocrité des productions dans le domaine des arts appliqués. Les réactions sont nombreuses : William Morris fonde sa société, à l'origine de la création en 1896 de la Central School of Arts and Crafts, dans laquelle des artistes participent pour la première fois à la conception et à la production d'articles d'usage quotidien. En Allemagne, c'est la naissance du Mouvement moderne qui place les critères de l'art industriel au-dessus de ceux de l'artisanat. À Weimar, en 1919, le Bauhaus intègre au sein de l'Académie écoles de métiers et de dessin industriel et 
élimine ainsi « le dogme funeste de l'art pour l'art », puisque cette école peut exercer à la fois une influence matérielle sur l'évolution de l'art aussi bien que sur l'industrie. En soulignant la supériorité éthique du designer sur le peintre solitaire et sans public, Pevsner défend l'avènement d'une complémentarité nouvelle entre l'art et l'industrie et signe donc là une œuvre engagée.

Mais l'intérêt de cet ouvrage est ailleurs, et on reste saisi par la qualité des intuitions de son auteur et son impressionnante érudition dont témoigne la richesse des notes de bas de page, et l'on ne peut s'étonner que cet instrument de travail irremplacé continue à ouvrir des pistes (ainsi Carl Goldstein, Teaching art. Academies and schools from Vasari to Albers, Cambridge, Cambridge University Press, 1996).

Charlotte GuichaRD 